\title{
Realising the potential of metabolomics
}

\author{
metabolomics is starting on its next golden age; one in which it will finally \\ emerge and be recognized for the significant benefit it can provide."
}

Keywords: Core Information for Metabolomics Reporting " database " metabolic profiling " metabolite assignment $=$ metabolome n metabolomics $n$ metabonomics $=$ phenome $=$ standard

Even though it has been developing over a number of decades, metabolomics (also termed metabolic profiling or metabonomics) still struggles with the challenges of the rapid and accurate assessment of metabolites and the understanding of the biological roles of each of the component parts of the metabolome. Since small metabolites can occur in a range of cell types, be perturbed by a variety of metabolic processes, and share a general interconnectedness across multiple metabolomes, drawing meaningful conclusions from such complexity has proven difficult. Having passed through a long period of growth in the optimization of techniques for sample analysis and the development of an array of data analysis tools, metabolomics has matured to a point where study aims are now focusing again on the original biological questions. To highlight what is changing is no longer an acceptable result; what must be determined is a view on the biological mechanism from the measured metabolite alterations. Addressing such challenges comes with an intrinsic dependency on open, ordered and searchable datasets, coupled with information to guide their application. Through the adoption of such a route, metabolomics could be in a position to make a larger and more significant impact to the advancement of biology; indeed to an extent comparable to that achieved through the genome.

The approaches used to measure the component parts of the metabolome represent different ends of a spectrum of analysis. At one end of the scale stands the open-profile approach typically used to determine the changes from which hypotheses can be drawn, whilst at the other end is the targeted assay, an example of the classical hypothesis testing paradigm. Over the development phase of metabolomics, each of these two approaches has been presented as the solution to metabolomic assessment. The outcome, and the strength for metabolomics, is that today a hybrid of the two provides the most objective of assessments, coupled with the ability to rapidly obtain information to answer specific questions in a timely manner. However, for the open part of this approach, the major bottleneck affecting metabolomics remains the identification of the small molecules measured in the analytical phase.

Small molecules can be difficult to accurately identify de novo since, unlike proteins and nucleic acids, many cannot be deconstructed into sets of common units, that is, amino acids or nucleotides, respectively. Even though the principal analytical approaches - LC, GC, NMR spectroscopy and MS - provide powerful tools for small-molecule investigation, identification can still be a protracted process. Utilization of characteristic information or patterns in data from MS-based metabolomics have enabled a more rapid and consistent characterization of the small-molecule components of a sample. However, to move metabolomics from the bench into the field or by the bedside, bioanalysts need to focus on the application of targeted assays, even if these have many analytes. Thus, identification is the cornerstone to further deployment of metabolomics solutions.

Although literature data contribute to the identification of these metabolites and the biological relevance, having more ordered repositories of data starts to reduce the expected variability we see between disconnected experiments. The metabolomics community benefits from a variety of online data/information sources that cover the composition of the metabolome and information on the biological involvement of each of the component molecules (e.g., the Human Metabolome Database [1,101], LIPID MAPS [102] and Chemical Entities of Biological Interest $[2,103])$, sources of analytical data with assignment to aid in identification (e.g., Biological Magnetic Resonance Data Bank [3,104], Birmingham Metabolite Library [4,105], MetLin [5,106] and Platform for Riken Metaoblomics [6,107]) and, most recently,

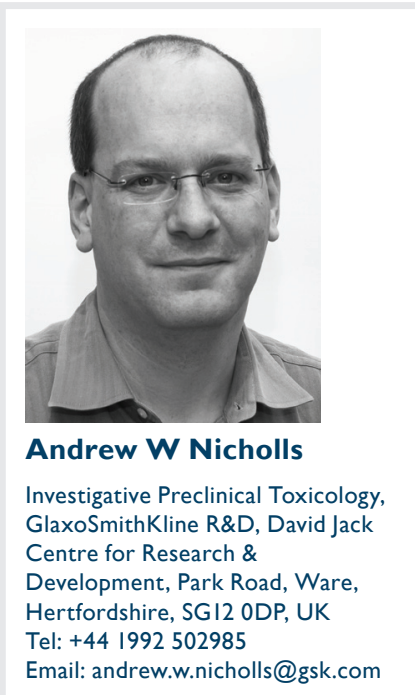

FUTURE
SCIENCE FSS 
repositories for the sharing of metabolomics experimental data such as MetaboLights [108] and XCMS Online [7,109]. However, these represent only part of a larger number of repositories, and an expected increase in the major and specialist data repositories will require a more central portal to connect them together, so as to maximize the value of the unified information. An example of this type of approach has come from the ChemSpider web resource, which links together many data repositories concerning chemical structure, naming and properties, and is now sponsored by the Royal Society of Chemistry [110]. Such a metabolomics portal would aid reliable assignment, providing physical data consistent with a molecule's structure, but also connect to information to determine the biological likelihood. Furthermore, connecting to databases of previous experiments where the molecule of interest may already have been assigned, in the form that it is found in the tissue or biological fluid, should provide a more accurate means of assignment. Metabolomics would benefit from such a resource, should a suitable sponsor arise.

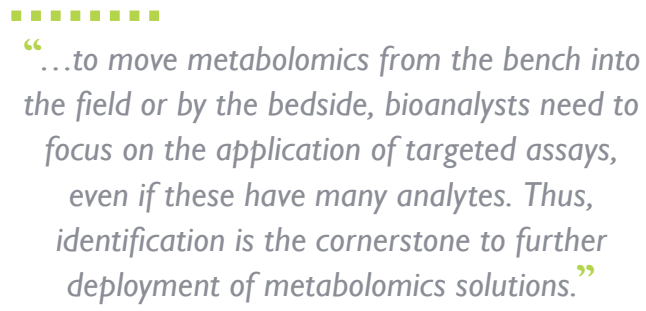

Even with the benefits that unified repositories can provide, they can still be affected by variability, in terms of the types of data, how the data were obtained, processed, stored, annotated and interpreted, as well as the variation in the original biological experiment. To solve this, the metabolomics community can at least turn to the work that has been done to date in standardizing data description and information. The establishment of the Core Information for Metabolomics Reporting, through the provision of a set of reporting standards covering the biological phase [8-11], chemical analysis [12], data analysis [13], ontologies [14] and exchange of raw data [15], was an early collaborative success bringing together previous efforts to deliver a common agreement. These standards have been incorporated into a number of online resources, including MetaboLights [108] and the Birmingham Metabolite Library [4,105], and adherence to them is now expected for submission to the relevant peer-reviewed journals. This regulation of work, with accurate recording of the conditions used, affords the opportunity to push forward the knowledge of metabolomics and its management.

Repositories of raw metabolomics experimental data provide a new opportunity for researchers to actively combine data to enhance data analysis via a community approach, or to explore the data for questions beyond the initial study aims. Coupling these with the growth of online tools for metabolomics data analysis/modeling, such as Metaboanalyst [16] and XCMS Online $[7,109]$, could result in studies that genuinely aim to look at metabolome studies at the level of the population and not simply the sample. Agreed standards of practice still need to be enforced to ensure that metabolomics does not become a victim of poorly validated statistics. Furthermore, connection of these tools and repositories will require an open approach to data sharing, whilst including the necessary safeguards to ensure protection of subjects where ethical and legal frameworks exist.

For such an approach, the usefulness of the data is critical and relies not only on capturing exactly what happened, but also on utilizing consistent approaches to the analysis of the samples and the original experimental design. Commonly used and tested approaches to sample analysis, including NMR spectroscopic [17] and MS approaches [18], are now available to provide guidance to the latest generation of metabolomics researchers, . Since the analytical phase has received much interest over the course of metabolomic development, it stands as a mature part of the workflow; yet enhancements will always still occur, necessitating their accurate reporting.

In comparison, the design of biological experiments still remains highly variable and there is a need for further optimization to minimize confounding factors that can affect the sensitivity of metabolomics. Efforts have started to influence the design of biological studies, but assessment of best practice still requires extensive work to balance metabolomics with ethical, environmental and biological factors. There is the opportunity for data repositories to also collate such study designs and provide community guidance to local practice.

Metabolomics has undergone a stream of development over the last two decades, which has enhanced and reinvented in equal measure. The original aim of providing a means to interrogate and understand the changes to the 
complete metabolome (the composite of each and every small molecule) was a bold intention when considering the complexity of the analysis and the interpretation. Those complexities still stand today, but through larger, managed collaboration these will be iteratively overcome. Indeed, collaboration is now becoming even more imperative with the formation of national centers and international projects that look to characterize the phenome via the metabolome [111]. Such work, utilizing the world's biobanks, will require stringency beyond that seen in earlier approaches, so that for both ethical and practical reasons, the maximum level of information can be obtained from such resources. With such work to come, it appears that metabolomics is starting on its next golden age; one in which it will finally emerge and be recognized for the significant benefit it can provide.

\section{Financial \& competing interests disclosure}

The author has no relevant affliations or financial involvement with any organization or entity with a financial interest in or financial conflict with the subject matter or materials discussed in the manuscript. This includes employment, consultancies, honoraria, stock ownership or options, expert testimony, grants or patents received or pending, or royalties.

No writing assistance was utilized in the production of this manuscript.

\section{References}

1 Wishart DS, Knox C, Guo AC et al. HMDB: a knowledgebase for the human metabolome. Nucl. Acids Res. 37, D603-D610 (2009).

2 de Matos P, Alcántara R, Dekker A et al. Chemical Entities of Biological Interest: an update. Nucl. Acids Res. 38(Suppl. 1), D249-D254 (2010).

3 Ulrich EL, Akutsu H, Doreleijers JF et al. BioMagResBank. Nucl. Acids Res. 36, D402-D408 (2007).

4 Ludwig C, Easton JM, Lodi A et al. Birmingham Metabolite Library: a publicly accessible database of 1-D ${ }^{1} \mathrm{H}$ and 2-D ${ }^{1} \mathrm{H}$ J-resolved NMR spectra of authentic metabolite standards (BML-NMR). Metabolomics 8(1), 8-18 (2012).

5 Smith CA, O'Maille G, Want EJ et al. METLIN: a metabolite mass spectral database. Ther. Drug Monit. 27(6), 747-751 (2005).

6 Akiyama K, Chikayama E, Yuasa $\mathrm{H}$ et al. PRIMe: a website that assembles tools for metabolomics and transcriptomics. In Silico Biol. 8(3-4), 339-345 (2008).

7 Tautenhahn R, Patti GJ, Rinehart D, Siuzdak G. XCMS Online: a web-based platform to process untargeted metabolomic data. Anal. Chem. 84(11), 5035-5039 (2012).

8 Griffin JL, Nicholls AW, Daykin CA et al. Standard reporting requirements for biological samples in metabolomics experiments: mammalian/in vivo experiments. Metabolomics 3, 179-188 (2007).

9 van der Werf MJ, Takors R, Smedsgaard $\mathrm{J}$ et al. Standard reporting requirements for biological samples in metabolomics experiments: microbial and in vitro biology experiments. Metabolomics 3, 189-194 (2007).

10 Fiehn O, Sumner LW, Rhee SY et al. Minimum reporting standards for plant biology context information in metabolomic studies. Metabolomics 3, 195-201 (2007).

11 Morrison N, Bearden D, Bundy JG et al. Standard reporting requirements for biological samples in metabolomics experiments: environmental context. Metabolomics 3, 203-210 (2007).

12 Sumner LW, Amberg A, Barrett D et al. Proposed minimum reporting standards for chemical analysis Chemical Analysis Working Group (CAWG) Metabolomics Standards Initiative (MSI). Metabolomics 3, 211-221 (2007).

13 Goodacre R, Broadhurst D, Smilde AK et al. Proposed minimum reporting standards for data analysis in metabolomics. Metabolomics 3, 231-241 (2007).

14 Sansone S, Schober D, Atherton HJ et al. Metabolomics standards initiative: ontology working group work in progress. Metabolomics 3, 249-256 (2007).

15 Hardy NW, Taylor CF. A roadmap for the establishment of standard data exchange structures for metabolomics. Metabolomics 3, 243-248 (2007).

16 Xia J, Mandal R, Sinelnikov I, Broadhurst D, Wishart DS. MetaboAnalyst $2.0-$ a comprehensive server for metabolomic data analysis. Nucl. Acids Res. 40(W1), W127W133 (2012).

17 Beckonert O, Keun HC, Ebbels TM et al. Metabolic profiling, metabolomic and metabonomic procedures for NMR spectroscopy of urine, plasma, serum and tissue extracts. Nat. Protoc. 2(11), 2692-2703 (2007).

18 Dunn WB, Broadhurst D, Begley P et al. Human Serum Metabolome (HUSERMET) Consortium. Procedures for large-scale metabolic profiling of serum and plasma using gas chromatography and liquid chromatography coupled to mass spectrometry. Nat. Protoc. 6(7), 1060-1083 (2011).

\section{- Websites}

101 Human Metabolome Database. www.hmdb.ca

102 The LIPID MAPS Lipidomics Gateway. www.lipidmaps.org

103 Chemical Entities of Biological Interest. www.ebi.ac.uk/chebi

104 Biological Magnetic Resonance Data Bank. www.bmrb.wisc.edu

105 Birmingham Metabolite Library. www.bml-nmr.org

106 METLIN: Metabolite and Tandem MS database. http://metlin.scripps.edu

107 Platform for Riken Metaoblomics. http://prime.psc.riken.jp

108 The MetaboLights Project. www.ebi.ac.uk/metabolights

109 XCMS Online. https://xcmsonline.scripps.edu

110 ChemSpider - the free chemical database. www.chempsider.com

111 MRC-NIHR Phenome Centre. www.mediacentre.dh.gov. uk/2012/08/01/a-phenomenal-legacy-forlondon-2012 